\title{
Moving Target Parameter Estimation and SFN Ghost Rejection in Multistatic Passive Radar
}

\author{
Yimin D. Zhang ${ }^{\dagger}$ and Braham Himed ${ }^{\ddagger}$ \\ $\dagger$ Center for Advanced Communications, Villanova University, Villanova, PA 19085, USA \\ $\ddagger$ Air Force Research Laboratory, AFRL/RYMD, Dayton, OH 45433, USA
}

\begin{abstract}
In this paper, we consider the motion parameter estimation of ground moving targets in a multistatic passive airborne radar that utilizes broadcast signals as a source of opportunity. It is shown, by focusing on the target position or within its close vicinity, that the narrow signal bandwidth permits the use of a moderate coherent integration time without range migration compensation. The multistatic nature, achieved through the use of multiple illumination sources, enables velocity and acceleration estimation in two-dimensional horizontal directions on the ground. A cost function is developed based on the estimated motion parameters which, when three or more illuminators are available, allows rejection of ghost targets in a single-frequency network (SFN) and proper parameter association in the presence of multiple closely spaced moving targets.
\end{abstract}

\section{INTRODUCTION}

In recent years, multistatic passive radars (MPRs) that use signals of opportunity attract significant interests because of many advantages, particularly due to their low-cost and covertness [1]-[6]. The fact that they do not emit wireless signals also makes them valuable for the reduction of frequency spectrum congestion.

Conventional active airborne radar systems typically operate in a monostatic mode with a wide signal bandwidth and a high power. In such a platform, motion parameter estimation of ground targets is an important problem applied in various surveillance applications. In particular, significant advances have been made to achieve sophisticated signal processing for motion parameter estimation, notably in the areas of timefrequency analysis, motion compensation, and range migration compensation (e.g., [7], [8]).

Multistatic passive radar systems, on the other hand, distinguish themselves with conventional active radars in a number of aspects, e.g., extremely narrow signal bandwidth, low signal power, bistatic operation, and the availability of multiple transmitters. In addition, passive radars may operate in a singlefrequency network (SFN), where identical waveforms are transmitted from multiple illuminators [1], [2], [9]. Literature in the area of multistatic passive radar systems and motion parameter estimation is still very limited (e.g., [4]-[6]).

In this paper, we investigate the problem of motion parameter estimation of ground moving targets in a multistatic

The work of Y. D. Zhang was supported in part by the Air Force Summer Faculty Fellowship Program under contract FA9550-09-C-0114, and by a subcontract with Dynetics, Inc. for research sponsored by the Air Force Research Laboratory (AFRL) under Contract FA8650-08-D-1303. passive airborne radar. Motion parameter estimation is very important to moving target indication and tracking as well as synthetic aperture radar (SAR) image enhancement. To address the unique features of the multistatic passive radar systems that distinguish conventional monostatic radar systems, the following issues are emphasized. 1) Because broadcast signals are not designed specifically for sensing, they are in general very weak and thus a long coherent processing interval (CPI), in an order of seconds, is often required to achieve reliable motion parameter estimation. As such, it is important to not only estimate the target velocity, but also the target acceleration. 2) Due to the bistatic nature, the Doppler frequencies observed in a passive radar are differently affected by the bistatic aspect angles of both the transmittertarget segment and the target-receiver segment. As such, the availability of multiple transmitters, which are typically well separated in space, permits unambiguous estimation of the full motion parameters in different directions. 3) With the extremely narrow bandwidth, the range migration problem appears to be very different to wideband systems. When a specific ground position is concerned, the dominant source of range migration due to the motion of the receive radar platform can be compensated for by a known reference range, and a moderate CPI can be used without the necessity of accounting for the range migration due to target motions. The use of Keystone transform, on the other hand, would permit an extended CPI without considering the target acceleration.

In digital audio/video broadcast (DAB/DVB) services operate in an SFN, a passive radar may suffer from the ghost target problem where the number of signal copies depends on the number of illuminators in the range [9]. While the ghost targets may not be easily distinguished from true targets from SAR images, they can be clearly separated by exploiting estimated motion parameters. In particular, for moving targets considered in a two-dimensional (2-D) ground space, a cost function is developed based on the estimated motion parameters to allow for ghost rejection when three or more illuminators are available. On the other hand, when multiple moving targets are closely located, estimation of their motion parameters requires their observed Doppler signatures to be properly associated. The same cost function can be used to achieve this goal.

The following notations are used in this paper. A lower (upper) case bold letter denotes a vector (matrix). $(\cdot)^{*},(\cdot)^{T}$ and $(\cdot)^{H}$ respectively denote complex conjugation, transpose, and conjugate transpose (Hermitian) operations. $\dot{a}=d a / d t$ (or $\dot{\mathbf{a}}=d \mathbf{a} / d t$ ) denotes the derivative of variable $a$ (or vector 
a) with respect to time. $\mathbf{I}_{N}$ expresses the $N \times N$ identity matrix, and blkdiag $(\cdot)$ denotes the operation to form a block diagonal matrix. In addition, $\|\cdot\|$ denotes the Euclidean norm of a vector.

\section{SignAL MODEL}

\section{A. Geographical Relationship}

We consider an MPR system where an airborne receiver moves along its track direction, which coincides with the $x$ axis, in a constant vector velocity of $\mathbf{v}_{r}$. We assume that $N$ stationary illuminators are available and their positions are known a priori.

Denote $\mathbf{r}(t)=\mathbf{r}_{0}+\mathbf{v}_{r} t$ as the trajectory of the receiver, where $\mathbf{r}_{0}$ is the initial position vector, and $\mathbf{v}_{r}$ is the velocity vector. The trajectory of the moving target is denoted as $\mathbf{p}(t)=\mathbf{p}_{0}+\mathbf{v} t+\mathbf{a} t^{2} / 2$ and the corresponding instantaneous velocity is $\dot{\mathbf{p}}(t)=\mathbf{v}+\mathbf{a} t$, where $\mathbf{p}_{0}, \mathbf{v}$, and $\mathbf{a}$ are, respectively, the initial target position, initial velocity, and acceleration vectors. All these parameters are defined in the three-dimensional (3-D) Cartesian coordinate. We assume that the target is located on the ground, i.e., the $z$-axis components of the target position, velocity, and acceleration are all zero. As a result, the four unknown parameters that characterize the motion of the target are defined in the $x$ - and $y$-axes as $\overline{\mathbf{v}}=\left[v_{x}, v_{y}\right]^{T}$ and $\overline{\mathbf{a}}=\left[a_{x}, a_{y}\right]^{T}$.

The range between the $i$ th illuminator, located at $\mathbf{t}^{i}, i=$ $1, \ldots, N$, and the receiver, which is located at $\mathbf{r}(t)$, is expressed as

$$
r^{i}(t)=\left\|\mathbf{r}(t)-\mathbf{t}^{i}\right\|
$$

and the radial velocity between them is obtained as

$$
\dot{r}^{i}(t)=\frac{\left[\mathbf{r}(t)-\mathbf{t}^{i}\right]^{T} \dot{\mathbf{r}}(t)}{\left\|\mathbf{r}(t)-\mathbf{t}^{i}\right\|} .
$$

On the other hand, the bistatic range between the $i$ th transmitter, the target, and the receiver, is expressed as,

$$
\rho^{i}(t)=\left\|\mathbf{p}(t)-\mathbf{t}^{i}\right\|+\|\mathbf{p}(t)-\mathbf{r}(t)\|,
$$

and the corresponding bistatic velocity is

$$
\dot{\rho}^{i}(t)=\frac{\left[\mathbf{p}(t)-\mathbf{t}^{i}\right]^{T} \dot{\mathbf{p}}(t)}{\left\|\mathbf{p}(t)-\mathbf{t}^{i}\right\|}+\frac{[\mathbf{p}(t)-\mathbf{r}(t)]^{T}[\dot{\mathbf{p}}(t)-\dot{\mathbf{r}}(t)]}{\|\mathbf{p}(t)-\mathbf{r}(t)\|}
$$

\section{B. Transmit and Receive Signals}

The $i$ th illuminator emits a narrowband bandpass waveform with analytic baseband representation $u^{i}(t)$. The reference channel signal, i.e., the direct path signal, received by the receiver from the $i$ th transmitter is given by

$$
s_{r}^{i}(t)=u^{i}\left(t-r^{i}(t) / c\right) e^{-j 2 \pi f_{c} r^{i}(t) / c}+n_{r}^{i}(t),
$$

where $c$ is the velocity of propagation, $f_{c}$ is the carrier frequency, and $n_{r}^{i}(t)$ is the additive noise. The subscript " $r$ " denotes the reference channel. The observation time is assumed to be $t=\left[0, \Delta_{s}, 2 \Delta_{s}, \ldots, T-\Delta_{s}\right]$, where $\Delta_{s}=$ $1 / f_{s}$ is the sampling interval corresponding to the sampling frequency $f_{s}$. The overall observation yields a total number of $T f_{s}$ samples.
In practice, it is reasonably assumed that the noise in the reference signal can be removed. This is justified by the fact that digital broadcast systems (e.g., DAB or DVB-T) use forward error correction (FEC) coding and thereby enable passive radar receivers to perfectly reconstruct the transmitted signal after successful demodulation [4], [10]. As such, the baseband direct path signal becomes

$$
s_{r}^{i}(t)=u^{i}\left(t-r^{i}(t) / c\right) e^{-j 2 \pi f_{c} r^{i}(t) / c} .
$$

On the other hand, the surveillance channel signal, i.e., the target-reflected signal, is given at the baseband by

$$
s_{s}(t)=\sum_{i=1}^{N} \sigma^{i} u^{i}\left(t-\rho^{i}(t) / c\right) e^{-j 2 \pi f_{c} \rho^{i}(t) / c}+n_{s}(t),
$$

where the subscript " $s$ " denotes the surveillance channel, $\sigma^{i}$ is the target reflectivity corresponding to the $i$ th illuminator, and $n_{s}(t)$ is the additive noise. It is assumed that the reference and the surveillance channels have been sufficiently conditioned to prevent significant leakage between these channels [6].

\section{Matched Filtering}

A segmented reference signal is used to correlate with the surveillance signal. It yields a sequence of matched filter output at time instants $t_{1}, \ldots, t_{M}$, where $M$ is the number of azimuth samples and $\Delta t=t_{m}-t_{m-1}$ is the azimuthal sampling interval. At the output of the matched filter, the phase term is determined by the difference between the transmittertarget-receiver bistatic range and the transmitter-receiver direct path distance. By considering the motion of the radar platform and the target, the range difference is expressed as

$$
\begin{aligned}
\rho^{i}\left(t_{m}\right)-r^{i}\left(t_{m}\right) & =\left\|\mathbf{p}_{0}+\mathbf{v} t_{m}+\mathbf{a} t_{m}^{2} / 2-\mathbf{t}^{i}\right\| \\
& +\left\|\mathbf{p}_{0}+\mathbf{v} t_{m}+\mathbf{a} t_{m}^{2} / 2-\mathbf{r}_{0}-\mathbf{v}_{r} t_{m}\right\| \\
& -\left\|\mathbf{r}_{0}+\mathbf{v}_{r} t_{m}-\mathbf{t}^{i}\right\| .
\end{aligned}
$$

Applying the Taylor series expansion to the above expression and ignoring insignificant high-order components, we obtain

$$
\begin{aligned}
& \rho^{i}\left(t_{m}\right)-r^{i}\left(t_{m}\right) \\
& \approx\left(\rho_{0}^{i}-r_{0}^{i}\right) \\
& +\left\{\frac{\left[\mathbf{p}_{0}-\mathbf{t}^{i}\right]^{T} \mathbf{v}}{\left\|\mathbf{p}_{0}-\mathbf{t}^{i}\right\|}+\frac{\left[\mathbf{p}_{0}-\mathbf{r}_{0}\right]^{T}\left(\mathbf{v}-\mathbf{v}_{r}\right)}{\left\|\mathbf{p}_{0}-\mathbf{r}_{0}\right\|}-\frac{\left[\mathbf{r}_{0}-\mathbf{t}^{i}\right]^{T} \mathbf{v}_{r}}{\left\|\mathbf{r}_{0}-\mathbf{t}^{i}\right\|}\right\} t_{m} \\
& +\left\{\frac{\left[\mathbf{p}_{0}-\mathbf{t}^{i}\right]^{T} \mathbf{a}}{\left\|\mathbf{p}_{0}-\mathbf{t}^{i}\right\|}+\frac{\left[\mathbf{p}_{0}-\mathbf{r}_{0}\right]^{T} \mathbf{a}}{\left\|\mathbf{p}_{0}-\mathbf{r}_{0}\right\|}+\frac{\left\|\mathbf{v}_{r}\right\|^{2}-2 \mathbf{v}_{r}^{T} \mathbf{v}_{x}}{\left\|\mathbf{p}_{0}-\mathbf{r}_{0}\right\|}-\frac{\left\|\mathbf{v}_{r}\right\|^{2}}{\left\|\mathbf{r}_{0}-\mathbf{t}^{i}\right\|}\right\} \frac{t_{m}^{2}}{2} .
\end{aligned}
$$

In the above expression, the first term,

$$
\rho_{0}^{i}-r_{0}^{i}=\left\|\mathbf{p}_{0}-\mathbf{t}^{i}\right\|+\left\|\mathbf{p}_{0}-\mathbf{r}_{0}\right\|-\left\|\mathbf{r}_{0}-\mathbf{t}^{i}\right\|,
$$

is the constant range difference term. The second term is related to range walk or the constant Doppler shift. The third term is related to the range curvature or linear Dopplerfrequency modulation.

\section{Range Migration Correction}

In wideband radar systems, because of the high range resolution, range migration must be compensated before coherent signal processing can be performed over the azimuthal time. 
In the underlying passive radar system, however, because of the narrow signal bandwidth and thus the low bistatic range resolution, the system is more tolerant to range migration than its wideband counterparts. In particular, by focusing on the target position or within its close vicinity, range migration due to the radar platform motion is inherently corrected. For the simplicity of description, the problem is considered in this paper without performing range migration compensation due to the moving target. It is pointed out, nevertheless, that compensating the range migration of the moving target through, e.g., the commonly used Keystone transform [7], would allow extended CPI for improved motion parameter estimations.

In (9), we notice that the dominant source of range migration is the radar platform, whose motion parameters are known. Therefore, we can compensate the range migration due to the motion of the radar platform for those terms that are not coupled with others. Because the range depends on a specific target position ( $\mathbf{p}_{0}$ in (9)), such compensation is only effective in the vicinity of the selected reference point.

Consider a ground reference position (which is also referred to as the scene origin) with a coordinate of $\mathbf{q}$. Then, the bistatic range between the $i$ th transmitter, the scene origin, and the receiver, is expressed as,

$$
\begin{aligned}
\zeta^{i}\left(t_{m}\right) & =\left\|\mathbf{q}-\mathbf{t}^{i}\right\|+\left\|\mathbf{q}-\mathbf{r}\left(t_{m}\right)\right\| \\
& \approx\left\|\mathbf{q}-\mathbf{t}^{i}\right\|+\left\|\mathbf{q}-\mathbf{r}_{0}\right\|+\frac{\left[\mathbf{q}-\mathbf{r}_{0}\right]^{T} \mathbf{v}_{r}}{\left\|\mathbf{q}-\mathbf{r}_{0}\right\|} t_{m}+\frac{\left\|\mathbf{v}_{r}\right\|^{2}}{\left\|\mathbf{q}-\mathbf{r}_{0}\right\|} t_{(11)}^{2} .
\end{aligned}
$$

The corresponding delay, $\zeta^{i}\left(t_{m}\right)$, will be used in the matched filtering to replace $r^{i}(t)$, yielding the time delay term to be expressed as

$$
\begin{aligned}
\rho^{i}\left(t_{m}\right)-\zeta^{i}\left(t_{m}\right)= & \left\|\mathbf{p}_{0}+\mathbf{v} t_{m}+\mathbf{a} t_{m}^{2} / 2-\mathbf{t}^{i}\right\| \\
& +\left\|\mathbf{p}_{0}+\mathbf{v} t_{m}+\mathbf{a} t_{m}^{2} / 2-\mathbf{r}_{0}-\mathbf{v}_{r} t_{m}\right\| \\
& -\left\|\mathbf{q}-\mathbf{t}^{i}\right\|-\left\|\mathbf{q}-\mathbf{r}_{0}-\mathbf{v}_{r} t_{m}\right\| .
\end{aligned}
$$

Let $\Delta=\mathbf{p}_{0}-\mathbf{q}$. Because the target is in the close vicinity of the scene origin, $\|\Delta\|$ is much smaller than the transmittertarget and the target-receive ranges, i.e., $\|\Delta\| \ll\left\|\mathbf{q}-\mathbf{t}^{i}\right\|$ for all $i$ and $\|\Delta\| \ll\left\|\mathbf{q}-\mathbf{r}_{0}\right\|$. Then, the above expression is approximated as

$$
\begin{aligned}
\rho^{i}\left(t_{m}\right)-\zeta^{i}\left(t_{m}\right) & \left(\mathbf{q}-\mathbf{t}^{i}\right)^{T} \Delta \\
& \approx \frac{\left(\mathbf{q}-\mathbf{r}_{0}\right)^{T} \Delta}{\left\|\mathbf{q}-\mathbf{t}^{i}\right\|}+\frac{\left(\mathbf{q}-\mathbf{r}_{0} \|\right.}{\left.\| \mathbf{q}-\mathbf{t}^{i}\right)^{T} \mathbf{v}} \\
& +\left\{\frac{\left(\mathbf{q}-\mathbf{r}_{0}\right)^{T} \mathbf{v}}{\left\|\mathbf{q}-\mathbf{t}^{i}\right\|}\right\} t_{m} \\
& +\left\{\frac{\left(\mathbf{q}-\mathbf{t}^{i}\right)^{T} \mathbf{a} \|}{\left\|\mathbf{q}-\mathbf{t}^{i}\right\|}+\frac{\left(\mathbf{q}-\mathbf{r}_{0}\right)^{T} \mathbf{a}}{\left\|\mathbf{q}-\mathbf{r}_{0}\right\|}-\frac{2 \mathbf{v}_{r}^{T} \mathbf{v}}{\left\|\mathbf{q}-\mathbf{r}_{0}\right\|}\right\} \frac{t_{m}^{2}}{2}
\end{aligned}
$$

As in (9), the terms in the right-hand side respectively represent the constant term, range walk, and range curvature. In a typical operation environment, the contribution of $\mathbf{v}_{r}$ is insignificant, and the range migration is primarily due to the target motion, allowing more than a second of CPI within which the target does not walk beyond half of the bistatic range resolution.

\section{Azimuthal Time Doppler Estimation And MOTION PARAMETER ESTIMATIONS}

\section{A. Azimuthal Time Doppler Estimation}

When the range migration is corrected, as described in the previous section, the signals corresponding to a target is aligned within the same range cell over a certain CPI. Then, time-frequency analysis techniques can be applied against the azimuthal time to analyze the instantaneous Doppler signature. The Doppler signatures after range migration are approximated as linear frequency modulated (LFM) signals, and their characteristics can be estimated using a number of techniques, such as the Radon-Wigner transform, the fractional Fourier transform (FrFT), and the chirp-Fourier transform (e.g., [12][15]). These techniques have been commonly applied in SAR systems and other radar applications (e.g., [16]-[18]). In this study, the Radon-Wigner transform is used.

\section{B. Motion Parameter Estimation}

In (13), we see that the unknown variables governing a moving target is the $x$ - and $y$-axis components of the target's velocity and acceleration, totaling 4 unknowns. On the other hand, the number of parameters we can estimate from each data set through the analysis of azimuthal data is 2 (Doppler centroid and chirp rate). Note that, because of phase wrapping, the phase information cannot be used for the estimation of the range displacement.

Denote $f_{0}^{[i]}$ and $\beta^{[i]}$ as the initial Doppler frequencies and the chirp rate corresponding to $\rho^{i}\left(t_{m}\right)-\zeta^{i}\left(t_{m}\right), m=$ $1,2, \ldots, M$. From (13), they are related to the target velocity and acceleration as

$$
\left[\begin{array}{l}
f_{0}^{[i]} \\
\beta^{[i]}
\end{array}\right]=\mathbf{A}^{i}(\mathbf{q})\left[\begin{array}{c}
\mathbf{v}_{0} \\
\mathbf{a}
\end{array}\right]
$$

where

$\mathbf{A}^{i}(\mathbf{q})=-\frac{1}{\lambda}\left[\begin{array}{cc}\frac{\left(\mathbf{q}-\mathbf{t}^{i}\right)^{T}}{\left\|\mathbf{q}-\mathbf{t}^{i}\right\|}+\frac{\left(\mathbf{q}-\mathbf{r}_{0}\right)^{T}}{\left\|\mathbf{q}-\mathbf{r}_{0}\right\|} & \mathbf{0} \\ -\frac{2 \mathbf{v}_{r}^{T}}{\left\|\mathbf{q}-\mathbf{r}_{0}\right\|} & \frac{\left(\mathbf{q}-\mathbf{t}^{i}\right)^{T}}{\left\|\mathbf{q}-\mathbf{t}^{i}\right\|}+\frac{\left(\mathbf{q}-\mathbf{r}_{0}\right)^{T}}{\left\|\mathbf{q}-\mathbf{r}_{0}\right\|}\end{array}\right]$ and $\lambda=c / f_{c}$ is the wavelength. Because of the involvement of the transmitter position $\mathbf{t}^{i}$, the use of $N$ spatially separated illuminators yields $2 N$ distinct equations, expressed in matrix format as

$$
\phi=\mathbf{A}(\mathbf{q}) \boldsymbol{\theta},
$$

where $\phi=\left[f_{0}^{[1]}, \beta^{[2]}, \cdots, f_{0}^{[N]}, \beta^{[N]}\right]^{T}, \mathbf{A}(\mathbf{q})=\left[\left(\mathbf{A}^{1}(\mathbf{q})\right)^{T}\right.$, $\left.\cdots,\left(\mathbf{A}^{N}(\mathbf{q})\right)^{T}\right]^{T}$, and $\boldsymbol{\theta}=\left[\mathbf{v}_{0}^{T}, \mathbf{a}^{T}\right]^{T}$. As a result, the 4 unknown motion parameters can be uniquely solved when $N \geq 2$. The use of 2 illuminators allows unambiguous motion parameter estimation, whereas more illuminators will yield an overdetermined problem for improved motion parameter estimation performance.

\section{SFN Ghost Rejection}

By broadcasting the same waveform in the same frequency band from multiple illuminators, SFN broadcasting achieves 
higher spectrum efficiency as compared to the multi-frequency network (MFN) counterparts. Because of the slow symbol rate used in coded OFDM (COFDM) schemes, signals transmitted from different transmitters act as frequency-nonselective fading and do not generate inter-symbol interference.

In an SFN with $N$ transmitters, a receiver receives $N$ copies of the signal from a single target. Due to lack of association information, the receiver does not know which one corresponds to which transmitter. As a result, all the $N$ copies of signal should be processed against the bistatic systems associated with the $N$ different transmitters, yielding $N^{2}$ bistatic configurations. Fig. 1 shows an example of a three-transmitter scenario, where the detailed parameters are described in the Section IV. These $N^{2}$ ellipses generate a number of cross-sections as potential ghosts.

Because the position of a ghost target is usually far separated from the true target position, the received signals do not yield meaningful Doppler parameter estimation because the range walking due to the radar receiver platform is not compensated. In addition, when three or more transmitters are available, motion parameter estimation allows ghost rejection from the following perspectives:

(a) Determine as a ghost when signals from only one or two transmitters are recognized.

(b) For a position with signals from all the available transmitters, because the motion parameter estimation problem is overdetermined, only parameters estimated at the true target positions yield small distances between them and the observation data.

(c) The estimated motion parameters at ghost positions may take values that are beyond the reasonable range.

The relation (16) holds for all the transmitters when the true target is considered. In this case, the error between $\phi$ and $\mathbf{A}(\mathbf{q}) \boldsymbol{\theta}$ is primarily due to noise and is thus very small. For ghost positions, on the other hand, there is no such intrinsic relationship between the observed Doppler signature and the motion parameters. Therefore, the motion parameters estimated from this expression are merely a mathematical solution that best fits this equation. As a result, we can expect a large difference between $\boldsymbol{\phi}$ and $\mathbf{A}(\mathbf{q}) \boldsymbol{\theta}$ in this case.

Therefore, we use the following criterion to accept or reject a position under test as a legitimate target or a ghost:

$$
\eta=\frac{\|\mathbf{P}[\boldsymbol{\phi}-\mathbf{A}(\mathbf{q}) \boldsymbol{\theta}]\|}{\|\mathbf{P} \phi\|}
$$

where $\mathbf{P}=\operatorname{blkdiag}\left[\mathbf{I}_{N}, T \mathbf{I}_{N}\right]$. We declare a legitimate target when $\eta \leq \eta_{0}$ and a ghost when $\eta>\eta_{0}$, where $\eta_{0}$ is a pre-determined threshold. Because the estimation error of the parameters is typically a small fraction of the parameters themselves, $\eta_{0}$ can be chosen to be much smaller than one.

\section{Parameter Association with Multiple Targets}

When multiple targets are closely spaced and appear in the same range bin for different bistatic settings, correct motion parameter estimation is achieved only when the target Doppler signatures corresponding to different illuminators are properly associated. Such situations are very likely to happen because of the narrow signal bandwidth used in passive radar systems. The Doppler frequency analysis can separate multiple targets in the time-frequency domain as long as their Doppler signatures are not identical. However, because these signatures appear in the same range, we obtain $K$ sets of parameters for each bistatic setting with a transmitter, where $K$ is the number of targets. For each parameter set, we need to identify the parameters that are associated with the same target in other bistatic settings.

The cost function expressed in (17) can be used to associate the target combinations in an overdetermined scenario, that is, 3 or more transmitters are available. Under the similar concept, when all the estimated Doppler signatures obtained from the same target, the cost function defined in (17) takes a small value. Otherwise, when the Doppler signatures belong to different targets, the cost function will take a large value.

\section{Simulation Results}

In the simulations, we use DAB signals as sources of opportunity. The DAB signals, which use COFDM, are specified in [11]. The main characteristics of a DAB signal are as follows: (1) The symbol has 1-ms useful duration with a guard interval of $0.246 \mathrm{~ms}$; (2) 1536 sub-carriers are transmitted simultaneously per symbol with a sub-carrier spacing of 1 $\mathrm{KHz}$, which yields an overall signal bandwidth of $1.537 \mathrm{MHz}$; and (3) the QPSK coding is used for each sub-carrier. The transmitters are assumed to be operated at a transmission frequency band around $230 \mathrm{MHz}$. Three broadcast stations are considered, whose coordinates are $[-12,10,0.1]^{T} \mathrm{~km},[15$, $-15,0.1]^{T} \mathrm{~km}$, and $[-12,-10,0.1]^{T} \mathrm{~km}$, respectively.

\section{A. Single Target in an SFN}

The coordinates of the initial position of the target is $\mathbf{p}_{0}=$ $[0,14,0]^{T} \mathrm{~km}$. Its initial velocity and acceleration are assumed to be $\mathbf{v}=[-15,-10,0]^{T} \mathrm{~m} / \mathrm{s}$ and $\mathbf{a}=[-1,-1,0]^{T} \mathrm{~m} / \mathrm{s}^{2}$, respectively. The reference point is chosen to be $\mathbf{q}=\mathbf{p}_{0}=$ $[0,14,0]^{T} \mathrm{~km}$.

The receive radar platform is assumed to move in the positive $x$-axis direction with a constant velocity of $150 \mathrm{~m} / \mathrm{s}$. The initial position of the radar platform is $[0,0,5]^{T} \mathrm{~km}$. The receiver data is sampled at a sampling rate of $2.048 \mathrm{MHz}$. We use half-overlapping segments, where the azimuthal position is sampled at $200 \mathrm{~Hz}$, and the correlation data length is $10 \mathrm{~ms}$. We assume that the transmitters are synchronized. A 1-second coherent processing time is used, which yields 200 azimuthal data samples. The input signal-to-noise ratio (SNR) is -45 dB.

Fig. 1 shows the total 9 ellipses corresponding to the 3 illuminator SFN. At the true target position $[0,14,0]^{T} \mathrm{~km}$, the initial velocity and acceleration estimates, in the $x$ - and $y$-axis directions, are respectively $\overline{\mathbf{v}}=[-14.84,-10.13]^{T}$ $\mathrm{m} / \mathrm{s}$ and $\overline{\mathbf{a}}=[-1.34,-0.70]^{T} \mathrm{~m} / \mathrm{s}^{2}$. The corresponding cost function is $\eta=0.0089$. On the other hand, the cross-sections of multiple ellipses yields possible ghosts. In particular, three ellipses cross around 2-D coordinates of $[-11.6,-16.6]^{T}$. At this position, one example of the estimated $2-\mathrm{D}$ velocity is $\overline{\mathbf{v}}=[-69.404,-7.578]^{T} \mathrm{~m} / \mathrm{s}$, and the acceleration is $\overline{\mathbf{a}}=$ 
$[10.587,-59.746]^{T} \mathrm{~m} / \mathrm{s}^{2}$. Note that both values are unusually high. The cost function, which is $\eta=0.528$ in this case, is much larger than 0.0089 for the true target position, enabling easy SFN ghost rejection when, say, $\eta_{0}=0.1$ is chosen. Note that a higher input SNR decreases the cost function at the true position, but generally not at a ghost position.

\section{B. Two Closely Spaced Targets}

Now we consider a scenario that, in addition to the target considered above, another target is added with an initial position of $[0.05,14,0]^{T} \mathrm{~km}$, i.e., 50 meters away from the first target. The initial ground velocity is $\overline{\mathbf{v}}_{0}=[-15,10]^{T}$ $\mathrm{m} / \mathrm{s}$, and the acceleration is $\overline{\mathbf{a}}=[1,1]^{T} \mathrm{~m} / \mathrm{s}^{2}$. The input SNR remains the same $-45 \mathrm{~dB}$ for all illuminators.

As illustrated in Fig. 2, two Doppler signatures are present corresponding to each illuminator, yielding two sets of chirp parameter estimates. These estimated parameter sets must be correctly associated to yield the correct motion parameter estimation. In Fig. 2(a), the circles are the estimated results for the first target with the correct association. In this case, the cost function is $\eta=0.0089$. On the other hand, Fig. 2(b) shows one example where the Doppler signatures are not correctly associated. In this case, a high value of $\eta=0.778$ is obtained, implying incorrect Doppler signature association.

\section{CONClusion}

In this paper, we have examined the motion parameter estimation of ground targets using an airborne passive radar that utilizes single-frequency network (SFN) broadcast signals as sources of opportunity. By focusing in the vicinity of the targets, the motion parameters are estimated based on the observed Doppler signatures corresponding to multiple illuminators. We also have developed a cost function which can be used to effectively determine whether a position with bistatic trajectory cross-sections corresponds to a legitimate target or an SFN ghost. The same cost function can also be used for target Doppler sigature association for correct motion parameter estimation when multiple moving targets are closely positioned.

\section{REFERENCES}

[1] H. D. Griffiths and N. R. W. Long, "Television-based bistatic radar," Proc. IEE - Radar Sonar Navig., vol. 133, no. 7, pp. 649-657, Dec. 1986.

[2] H. D. Griffiths and C. J. Baker, "Passive coherent location radar systems. Parts 1 \& 2," Proc. IEE - Radar Sonar Navig., vol. 152, no. 3, pp. 153168, Jun. 2005.

[3] G. Li, J. Xu, Y.-N. Peng, and X.-G. Xia, "Bistatic linear array SAR for moving target detection, location and imaging with two passive airborne radars," IEEE Trans. Geosci. Remote Sens., vol. 45, no. 3, pp. 554-565, March 2007.

[4] C. Berger, B. Demissie, J. Heckenbach, P. Willett, and S. Zhou, "Signal Processing for Passive Radar Using OFDM Waveforms," IEEE J. Selected Topics in Signal Proc., vol. 4 , no. 1, pp. 226-238, Feb. 2010.

[5] L. Wang, C. E. Yarman, and B. Yazici, "Doppler-hitchhiker: a novel passive synthetic aperture radar using ultranarrowband sources of opportunity," IEEE Trans. Geoscei. Remote Sens., vol. 49, no. 10, pp. 35213537, Oct. 2011.

[6] D. E. Hack, L. K. Patton, A. Kerrick, M. A. Saville, "Direct Cartesian Detection, Localization, and De-Ghosting for Passive Multistatic Radar," in Proc. IEEE Sensor Array and Multichannel Signal Processing Workshop, Hoboken, NJ, June 2012.
[7] R. P. Perry, R. C. DiPietro, R. L. Fente, "SAR imaging of moving targets," IEEE Trans. Aerosp. Electron. Syst., vol. 35, no. 1, pp. 188-200, Jan. 1999.

[8] F. Zhou, R. Wu, M. Xing, and Z. Bao, "Approach for single channel SAR ground moving target imaging and motion parameter estimation," IET Radar Sonar and Navig., vol. 1, no. 1, pp. 59-66, 2007.

[9] M. Daun and C. R. Berger, "Track initialization in a multistatic DAB/DVB-T network," in Proc. Int. Conf. Inform. Fusion, Cologne, Germany, June-July 2008.

[10] D. Poullin, "Passive detection using digital broadcasters (DAB, DVB) with COFDM modulation," IEE Proc. Radar, Sonar Navig., vol. 152, no. 3, pp. 143-152, June 2005.

[11] Radio Broadcasting Systems; Digital Audio Broadcasting (DAB) to Mobile, Portable and Fixed Receivers, Eur. Telecommun. Standards Inst., ETS-300-401, 1997.

[12] A. C. McBride and F. H. Kerr, "On Namias's fractional Fourier transform," IMA J. Applied Math., vol. 39, pp. 159-175, 1987.

[13] L. B. Almeida, "The fractional Fourier transform and time-frequency representations," IEEE Trans. Signal Proc., vol. 42, no. 11, pp. 30843091, Nov. 1994

[14] L. Cohen, Time-Frequency Analysis. Englewood Cliffs, NJ: PrenticeHall, 1995.

[15] X-G. Xia, "Discrete chirp-Fourier transform and its application to chirp rate estimation," IEEE Trans. Signal Proc., vol. 48, no. 11, pp. 3122-3133, Nov. 2000.

[16] S. Barbarossa and A. Farina, "Detection and imaging of moving objects with synthetic aperture radar. Part 2: Joint time-frequency analysis by Wigner-Ville distribution," IEE Proc. Pt. F, vol. 139. no. 1, pp. 89-97, 1992.

[17] V. C. Chen and H. Ling, Time-Frequency Transforms for Radar Imaging and Signal Analysis. Boston, MA: Artech House, 2002.

[18] H-B. Sun, G-S. Liu, H. Gu, and W-M. Su, "Application of the fractional Fourier transform to moving target detection in airborne SAR," IEEE Trans. Aerosp. Electron. Syst., vol. 38, no. 4, pp. 1416- 1424, Oct. 2002.

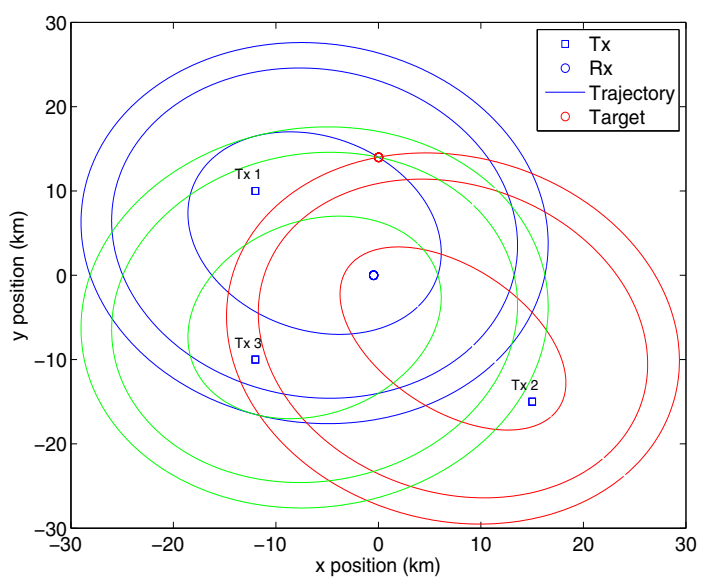

Fig. 1. Ellipses showing possible bistatic ranges due to SFN signaling.

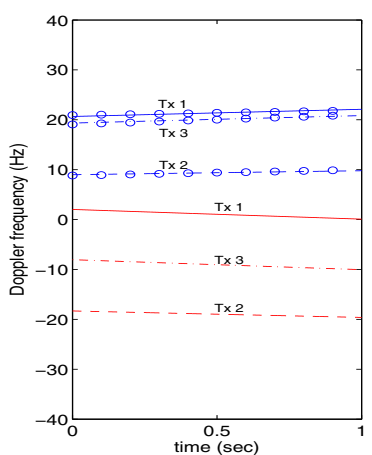

(a) Correct association

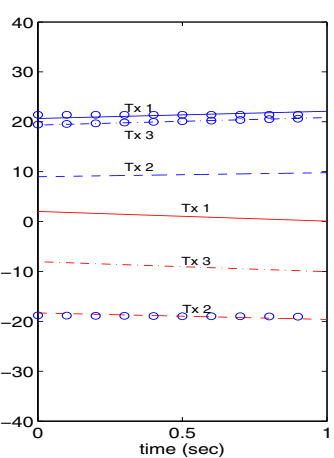

(b) Incorrect association
Fig. 2. Doppler signature association results in a two-target scenario. 